\title{
Rotation shadowing properties of circle and annulus maps
}

\author{
MARCY BARGE AND RICHARD SWANSON \\ Department of Mathematical Sciences, Montana State University, \\ Bozeman, MT 59717, USA
}

(Received 5 July 1987 and revised 20 December 1987)

\begin{abstract}
We define the notions of the pseudo-rotation set and rotation shadowing of pseudo-orbits for endomorphisms of the circle and for homeomorphisms of the annulus. The results include: the rotation shadowing property holds for all endomorphisms of the circle; the pseudo-rotation set equals the closure of the rotation set; the closure of the rotation set varies upper-semicontinuously.
\end{abstract}

\section{Introduction}

Roughly speaking, whenever there is a circle action on a manifold, one may define the rotation number of an orbit of a continuous endomorphism of the manifold. For example, one might wish to compute rotation numbers of a surface homeomorphism relative to a fixed point in the interior of an invariant disc. Except in the most idealized of situations, however, there is a spectrum of rotation numbers - a rotation interval - associated with a given orbit. In this article we are interested in the following problems:

(I) When is the rotation behavior of pseudo-orbits 'shadowed' or uniformly approximated by true rotation numbers? Can rotation sets be effectively calculated?

(II) Under what circumstances is the rotation set connected or closed? Is every irrational number in the rotation set attained as the actual limit of rotation averages?

That the first problem is not evident may surprise some - there is a sense that computers can safely calsulate rotation numbers except for roundoff error. However, one can find simple diffeomorphisms on the annulus for which pseudo-orbits with arbitrarily short jumps do not rotationally shadow true orbits (see definitions below). We shall, however, be able to rule out such behavior for circle maps. While for circle maps problem II is completely answered by work in [N-P-T] and [I], little is known about homeomorphisms on the annulus. It is our aim in this paper to demonstrate that pseudo-orbits, in addition to their intrinsic interest, shed light on the actual dynamics and rotation behavior of annulus maps. We should add, here, that Franks [F] uses pseudo-orbits to obtain information about the existence of periodic orbits in chain-transitive sets, and we shall make use, in $\S 3$, of one of his results. 
We shall prove the following results in this paper (definitions follow below):

(A) All continuous circle endomorphisms have the rotation shadowing property and the rotation set equals the pseudo-rotation set. (Theorem (1.1))

(B) For positive-tilt diffeomorphisms the rotation set equals the pseudo-rotation set. (Corrollary (3.10))

(C) Given any homeomorphism of the annulus, the closure of the rotation set equals the pseudo-rotation set. (Theorem (3.2))

Note that in particular the rotation set equals the pseudo-rotation set if and only if the rotation set is closed. Finally, using pseudo-orbits we can prove

(D) The closure of the rotation set depends upper-semicontinuously on the full homeomorphism group of the annulus, with continuity holding for a generic set of homeomorphisms. (Theorem (3.14))

\subsection{Definitions and terminology}

In the following discussion let $M$ denote a circle or annulus with covering spaces, respectively, $\tilde{M}=R$ or $R \times[0,1]$. Let $f: M \rightarrow M$ denote either a continuous endomorphism of the circle into itself or a homeomorphism of the annulus. In some cases we shall remark how our results apply to toral homeomorphisms. Let $F$ be a continuous lift of $f$ defined on the covering space $\tilde{M}$ of $M$. Let $\pi_{M}$ denote the covering map onto $M$, and let $\pi$ be the projection of the space $R \times[0,1]$ onto $R$. Define the rotation interval (wih respect to $F$ ) of $z$ to be the closed interval

$$
\rho(z)=\left[\lim _{n} \inf \left\{\pi F^{n}(\tilde{z}) / n\right\}, \limsup _{n}\left\{\pi F^{n}(\tilde{z}) / n\right\}\right] .
$$

The point $\tilde{z}$ is a lift of $z$; i.e. $\pi_{M}(\tilde{z})=z$. If $\rho(z)$ is a singleton set, it will be called a rotation number; otherwise, elements of $\rho(f)$ are called rotation elements. The union of rotation intervals over all $z$ in $M$ is called the rotation set of $f$, and is denoted by $\rho(f)$ or $\rho(F)$ if we have a particular lift in mind. Since $\rho(f)$ is well-defined up to integer translation, it is customary to set it equal to $\rho(F) \bmod 1$.

An $\alpha$-pseudo-orbit for a continuous mapping is an infinite sequence $\left\{p_{i}\right\}$ such that for a fixed metric $d($,$) , one has d\left(f\left(p_{i}\right), p_{i+1}\right) \leq \alpha$ for all $i$ in the sequence. By a well known result due to Bowen, in an Axiom $A$ system given any $\beta>0$, there exists an $\alpha>0$ such that every $\alpha$-pseudo-orbit is uniformly approximated (up to $\beta$ ) by the actual orbit of some point. Bowen called this ' $\beta$-shadowing of $\alpha$-pseudo-orbits' [Bo]. Pseudo-orbits are true orbits of nearby $\left(C^{0}\right.$-sense) diffeomorphisms. Pseudoorbits may also be viewed as the orbits generated via a computer simulation.

By adapting Bowen's terminology to rotation numbers, it is natural to query whether the average rotation of pseudo-orbits is $\beta$-shadowed by actual rotation numbers. In what follows, we restrict our attention to forward-infinite pseudo-orbits. We may suppose that $\left\{\tilde{z}_{i}\right\}$ denotes the lift of an $\alpha$-pseudo-orbit $\left\{z_{i}\right\}$ and is, in turn, the $\alpha$-pseudo-orbit of a fixed lift $F$ of $f$ defined on the covering space. Now define the pseudo-rotation interval by ( $\pi$ denotes projection onto the lift coordinate of the covering space)

$$
\rho\left(\tilde{z}_{i}\right)=\left[\lim _{i} \inf \pi \tilde{z_{i}} / i, \lim \sup _{i} \pi \tilde{z}_{i} / i\right]
$$


If $\left\{z_{i}\right\}$ is a true orbit with $z_{i}=f^{i}(z)$ for $i=1,2, \ldots$, then the latter definition gives the rotation interval $\rho(z)$ of $z$.

Now define $\rho(F, \alpha)$ to be the union of all rotation intervals of $\alpha$-pseudo-orbits. Finally, the pseudo-rotation set of $F$ is given by

$$
\rho_{\psi}(F)=\bigcap_{\alpha} \rho(F, \alpha) \text {. }
$$

If, in the preceding definition, we replace pseudo-orbits with true orbits we obtain $\rho(F)$, the actual rotation set of $F$. Analogously to the case of true rotation numbers, let the pseudo-rotation set of $f$ be given by $\rho_{\psi}(f)=\rho_{\psi}(F) \bmod 1$. Also, we say that the map $f \beta$-rotation-shadows pseudo-orbits if for each $\beta>0$, there exists $\alpha>0$ such that for every $\alpha$-pseudo-orbit $\left\{z_{n}\right\}$, with lift $\left\{\tilde{z}_{n}\right\}$, there exists an orbit $\left\{f^{n}(z)\right\}$, lifting to $\left\{F^{n}(\tilde{z})\right\}$, such that

$$
\lim \sup \left\{\left|F^{n}(\tilde{z})-\tilde{z}_{n}\right| / n\right\} \leq \beta .
$$

If $f \beta$-rotation-shadows pseudo-orbits for each $\beta>0$, we shall say that $f$ satisfies the rotation shadowing property. Note that one could replace $\beta$ by 0 in the definition and have a very strong rotation shadowing property that would be difficult to distinguish from the Bowen-Ruelle orbit shadowing. In any event our definition is more in the spirit of the shadowing lemma. Quite clearly, Axiom A systems satisfy the rotation shadowing property. Another kind of rotation-shadowing is to require that $\rho(f)=\rho_{\psi}(f)$, which is arguably a minimal necessary condition required to reliably calculate rotation sets. We have no examples of annular homeomorphisms which do not satisfy this weaker shadowing property, a property which can fail easily for toral homeomorphisms. In the next section we take up endomorphisms of the circle where a complete description of the rotation behavior is possible: all circle maps enjoy the rotation shadowing property. In $\S 2$ we apply ergodic theory to obtain detailed information about the structure of the rotation set, in particular the subset of actual rotation numbers obtained as limits of rotation averages. Finally, in the last section we prove, as our main result, that for annular homeomorphisms the closure of the rotation set equals the pseudo-rotation set.

\section{Endomorphisms of $S^{1}$}

For endomorphisms of $S^{1}$ having degree one, it is known that $\rho(f)$ is a closed interval ([N-P-T]) and, remarkably, that every closed subinterval of $\rho(f)$ is realized as the rotation set of some orbit $([\mathbf{I}]$ and $[\mathbf{B}-\mathbf{M}-\mathbf{P}-\mathbf{T}])$. The so-called standard family provides an important example of such endomorphisms and is given by $F_{\omega, \varepsilon}(z)=$ $z+2 \pi \omega+\varepsilon \sin 2 \pi z$. In one dimension we can prove the following:

THEOREM 1.1. All continuous circle endomorphisms of degree one have the rotation shadowing property.

Corollary 1.2. For all continuous maps $f: S^{1} \rightarrow S^{1}$

$$
\rho_{\psi}(f)=\rho(f) \text {. }
$$

That is, the rotation set equals the pseudo-rotation set and pseudo-orbits are a reliable guide to the true rotation behavior. 
Proof. We shall suppose that $F: R \rightarrow R$ is a continuous lift of $f$ such that $F(\tilde{z}+1)=$ $F(\tilde{z})+1$ for all $\tilde{z}$ in $R$. Before proving the theorem we need to establish three lemmas:

LEMMA 1. Let I denote a closed interval of length one in $R$, and let $N$ be a positive integer. Then given $\tilde{z} \in I$, there is a closed subinterval $J$ of $I$ such that $\tilde{z} \in J$ and length $\left(F^{N}(J)\right)=1$.

Proof of (1). Clearly, we have length $\left(F^{N}(I)\right) \geq 1$. Let $J=[\alpha, \beta]$, where $I=[c, d]$, $c \leq \alpha \leq \tilde{z}$ and $\tilde{z} \leq \beta \leq d$. Since the length of $F^{N}(J)$ depends continuously on the ordered pair $(\alpha, \beta)$, the lemma must follow from the intermediate value theorem.

LeMmA 2. Given $\alpha>0$, define $N(\alpha)$ to be the largest integer $k$ such that if $\left\{\tilde{z}_{n}\right\}$ is an $\alpha$-pseudo-orbit of $F$ then $\left|F^{n}\left(\tilde{z}_{0}\right)-\tilde{z}_{n}\right| \leq 1$ for $n=0,1,2, \ldots, k$. Then $N(\alpha)$ increases without bound as $\alpha$ tends to zero.

Proof of (2). This is simply the uniform continuity of $F$.

LEMMA 3. For any pair of positive integers $k$ and $N$, there exists a constant $C$ independent of $k$ such that the following holds:

$$
\sup \left\{\left|F^{r}(\tilde{z})-F^{r}(\tilde{w})\right|: 0 \leq r \leq N-1,|\tilde{z}-\tilde{w}| \leq k\right\} \leq C+k .
$$

Proof of (3). This result follows from the uniform continuity of $F$ and the fact that $F^{r}(\tilde{z}+k)=F^{r}(\tilde{z})+k$, for all $k$ and all $\tilde{z}$.

We are now ready to prove Theorem 1.1: Choose $N=N(\alpha)$ as in Lemma 2, fix any $\alpha$-pseudo-orbit $\left\{\tilde{z}_{n}\right\}$, and let $I$ be any interval of length 1 with $\tilde{z}_{0} \in I$. By Lemma 1 , choose an interval $J_{1}$ in $I$ such that $\tilde{z}_{0} \in J_{1}$ and length $\left(F^{N}\left(J_{1}\right)\right)=1$. By the choice of $N$, there is a number $l_{1} \in\{-1,0,1\}$ such that $\tilde{w}(1)=\tilde{z}_{N}+l_{1}$ lies in the interval $F^{N}\left(J_{1}\right)$. Note that whenever $\tilde{z}_{n}$ is an $\alpha$-pseudo-orbit, then so is $\tilde{z}_{n}+l$ for each integer $l$.

Now define, inductively, a sequence of intervals $\left\{J_{k}\right\}$ and a sequence of numbers $l_{k} \in\{-1,0,1\}$ as follows:

Given $J_{k}$ and $l_{k}$ with $\tilde{w}(k)=\tilde{z}_{k N}+\left(l_{1}+l_{2}+\cdots+l_{k}\right) \in J_{k}$, use Lemma 1 to obtain an interval $J_{k+1}$ such that $\tilde{w}(k) \in J_{k+1}$ and length $\left[F^{N}\left(J_{k+1}\right)\right]=1$. Now let $l_{k+1} \in$ $\{-1,0,1\}$ be such that $\tilde{w}(k+1)=\tilde{z}_{(k+1) N}+\left(l_{1}+\cdots+l_{k}\right)+l_{k+1}$ lies in $F^{N}\left(J_{k+1}\right)$. The latter choice makes use of Lemma 2.

By construction, $F^{N}\left(J_{k}\right)$ contains $J_{k+1}$ for each positive integer $k$. It follows that there exists an element $\tilde{z}$ in the set obtained by intersecting all the sets $F^{-k N}\left(J_{k+1}\right)$ for $k=0,1,2, \ldots$ We shall prove now that $F^{n}(\tilde{z})$ is the desired shadowing orbit.

We estimate as follows, where $n=k N+r$ and $N=N(\alpha)$ :

$$
\begin{aligned}
\left|F^{n}(\tilde{z})-\tilde{z}_{n}\right| & \leq\left|F^{r}\left(F^{k N}(\tilde{z})\right)-F^{r}\left(\tilde{z}_{k N}\right)\right|+\left|F^{r}\left(\tilde{z}_{k N}\right)-\tilde{z}_{k N+r}\right| \\
& \leq\left|F^{r}\left(F^{k N}(\tilde{z})\right)-F^{r}\left(\tilde{z}_{k N}\right)\right|+1(\text { Lemma 2) } \\
& \leq \mid F^{r}\left(F^{k N}(\tilde{z})\right)-F^{r}(\tilde{w}(k))+(C+k)+1(\text { Lemma 3) } \\
& \leq(C+1)+(C+k)+1 .
\end{aligned}
$$

Recalling that $n=k N+r$, it follows that

$$
\lim _{n} \sup \left\{\left|F^{n}(\tilde{z})-\tilde{z}_{n}\right| / n\right\} \leq 1 / N \text {. }
$$


Now, given $\beta>0$ choose $\alpha>0$ so that $1 / N=1 / N(\alpha)<\beta$, which is possible since $N(\alpha) \rightarrow \infty$, as $\alpha \rightarrow 0$.

Proof of Corollary 1.2. Since $f$ has the rotation shadowing property, $\rho(f)$ is dense in $\rho_{\psi}(f)$. But $\rho(f)$ is closed $([\mathrm{I}])$, so $\rho_{\psi}(f)=\rho(f)$.

\section{Ergodic theory and rotation sets}

Ergodic theory informs us that there is a close correspondence between time averages and space averages. Thus, one might expect to be able to realize rotation numbers as integrals with respect to invariant measures. Let $M_{f}(A)$ denote the set of $f$ invariant Borel probability measures defined on the annulus $A$. Recall that a measure is $f$-invariant if $\mu\left[f^{-1}(E)\right]=\mu[E]$, for all Borel sets $E$, and is ergodic if the only invariant measurable sets have measure one or zero. Given a fixed lift $F$ of the homeomorphism $f$ on the annulus, define a continuous function $g: A \rightarrow R$ such that

$$
g=\pi \circ(F-I d) \circ \sigma,
$$

where $\sigma: A \rightarrow R \times I$ denotes a cross-section of the covering map $\pi_{A}$ with range in the unit square of $R \times I$.

Given an $f$-invariant set $Z$ in $A$, let $M_{f}(Z)$ denote the set of all $f$-invariant Borel probability measures on $Z$. If $S$ is a subset of $R$ we denote the convex hull of $S$ by $\langle S\rangle$. The main theorem of this section is the following:

TheOREM 2.5. Let $Z$ denote any compact invariant subset of $A$. Let $\rho(Z)$ denote the rotation set obtained by restricting $f$ to $Z$. Then

(i) The supremum and infimum of $\rho(Z)$ are realized as rotation numbers of points in $Z$.

(ii) $\langle\rho(Z)\rangle=\left\{r=\int g(z) d \mu: \mu \in M_{f}(Z)\right\}$.

Towards the proof of this theorem we begin with the following:

Proposition 2.1. Given any ergodic measure $\mu$ on $A$ with respect to $f$, then the integral

$$
r=\int g(z) d \mu
$$

is the rotation number of some point in $A$. In fact, for $\mu$-almost all $w \in A$

$$
r=\lim _{n}\left\{F^{n}(\tilde{w}) / n\right\} \text {. }
$$

Remark. The integral in the latter proposition is called the mean rotation number with respect to $\mu$.

Corollary 2.2. The collection of $z \in A$ for which $\rho(z)$ is a rotation number is a set of full measure; that is, a set of measure one for every f-invariant probability measure.

Proof. This is essentially the ergodic theorem. Applying the latter, we infer that for almost all $w$ in the support of $\mu$,

$$
r=\lim _{n}\left\{\sum_{k=1}^{n} g\left(f^{k}(w)\right) / n\right\} .
$$


Using the periodicity of $F-I d$, it is not hard to show that

$$
g \circ f^{k}=\pi \circ F^{k+1} \circ \sigma-\pi \circ F^{k} \circ \sigma,
$$

and the desired result follows.

Remark. Following the language of ergodic theory we could call the set of such points $w \in A$ satisfying the proposition the generic points $G_{\mu}$ with respect to $\mu$. The set of points that are generic with respect to some measure are called the quasiregular points of $f$. It is well known that the quasiregular points have measure one for every invariant measure on $A$. See, for example [D-G-S, pp. 20-22].

Another proposition which is a standard application of ergodic theory is:

PROPOSITION 2.3. Let $\mu$ be any invariant probability measure on $A$. Then if

$$
g^{*}(z)=\lim _{n}\left\{\sum_{k=1}^{n} g\left(f^{k}(z)\right) / n\right\},
$$

$g^{*}$ is defined for $\mu$-almost all points and $\int_{A} g^{*}(z) d \mu=\int_{A} g(z) d \mu$. The range of $g^{*}$ consists entirely of rotation numbers.

Remark. The proposition holds for any compact invariant set in place of the annulus $A$.

The next proposition is familiar to ergodic theorists ([D-G-S]) and follows from the Banach-Alaoglu theorem. Recall that the weak*-topology on the space of measures is metrizable and has the pleasant property that a sequence $\mu_{n} \rightarrow \mu$ if and only if $\int h(z) d \mu_{n} \rightarrow \int h(z) d \mu$ for all continuous functions $h$ on $A$ (e.g. [D-G-S]). The annulus $A$ can be replaced by any compact invariant set $Z$ with Borel measures restricted to $Z$.

Proposition 2.4. Let $Z$ denote any compact invariant subset of $A$. In the weak*topology on the space of Borel measures on $Z, M_{f}(Z)$ is compact.

Proof of Theorem 2.5. (i) The evaluation mapping $E_{g}: \mu \rightarrow \int g(z) d \mu$ is obviously continuous in the weak*-topology on the set of Borel measures on $Z$. Thus, the mapping $E_{g}$ attains its infimum and supremum on the subset $M_{f}(Z)$ by (2.4). We shall first show that if $b=\sup \rho(Z)$, then for each $\varepsilon>0$ there exists $\mu \in M_{f}(Z)$ such that $b-\varepsilon<E_{g}(\mu)$. Later in the proof we will see that $E_{g}(\mu) \leq b$ for all $\mu \in M_{f}(Z)$. This will establish (i) since the proof for the infimum is similar. For any such $\varepsilon>0$, we may assume there exists an element $r$ of $\rho(Z)$ such that $b-\varepsilon<r \leq b$. It follows that $b-\varepsilon<\lim \sup _{n}\left\{\sum_{k} g\left(f^{k}(z)\right) / n\right\} \leq b$, for some point $z \in Z$.

Now use the method of Banach limits (e.g.; [R, p. 82]). Given any continuous function $h: Z \rightarrow R$ and $z \in Z$, as above, we shall define the bounded sequence

$$
z(h, n)=\sum_{k=1}^{N} h\left(f^{k}(z)\right) / n .
$$

By the Hahn-Banach Theorem, there exists a continuous linear functional $\Lambda: l^{\infty} \rightarrow R$ such that for all sequences $\{y(n)\} \in l^{\infty}$,

(a) $\Lambda\{z(g, n)\}=\sup \rho(z)$

(b) $\Lambda\{w(n)\}=\Lambda\{y(n)\}$, if $w(n)=y(n+1)$, and

(c) $\lim \inf \{y(n)\} \leq \Lambda\{y(n)\} \leq \lim \sup \{y(n)\}$. 
Now define the continuous linear functional $\mu(h)=\Lambda\{z(h, n)\}$. This is a signed $f$-invariant (by $(b)$ ) Borel measure which must be positive, since it is positive on positive functions. Such a measure satisfies the desired inequality when evaluated. Now suppose $E_{g}(\mu)=b$. Let $g^{*}$ be as in Proposition 2.3. Then $b=\int g^{*} d \mu$, where $\mu$ is supported on $Z$. Since no value of $g^{*}$, off a set of measure zero, can be larger than $b$, it must attain the constant value $b$ on a set of measure one (relative to $\mu$, of course). Note that this does not imply that $\mu$ is ergodic. The proof of (ii): Since both $a=\inf \langle\rho(Z)\rangle$ and $b=\sup \langle\rho(Z)\rangle$ are in the range of $E_{g}$ and $M_{f}(Z)$ is connected, $\langle\rho(Z)\rangle \subset\left\{r=\int g(z) d \mu: \mu \in M_{f}(Z)\right\}$. On the other hand, $a \leq g^{*}(z) \leq b$ for $\mu$-almost all $z \in Z$. By (2.3), then, $\left\{r=\int g(z) d \mu: \mu \in M_{f}(Z)\right\} \subset\langle\rho(Z)\rangle$.

\section{Homeomorphisms of $A$}

In this section we consider homeomorphisms of the annulus that are isotopic to the identity. Our main result (Theorem 3.2) asserts that, for such homeomorphisms, the closure of the rotation set is equal to the pseudo-rotation set. We also prove (Theorem 3.14) that the function which assigns to such a homeomorphism the closure of its rotation set is upper-semicontinuous. A key ingredient in our development is the following result due to John Franks.

THEOREM 3.1. (J. Franks [F].) Let $f: A \rightarrow A$ be an annulus homeomorphism isotopic to the identity. If $Z$ is a compact invariant chain transitive set in $A$ then every rational in $\langle\rho(Z)\rangle$ is the rotation number of a periodic orbit of $f$.

THEOREM 3.2. Let $f: A \rightarrow A$ be an annulus homeomorphism isotopic to the identity. Then $\rho_{\psi}(f)=\operatorname{cl}(\rho(f)$.

The above theorem is valid for circle endomorphisms (Corollary 1.2) but is far from being correct on the torus. Rotation vectors and pseudo-rotation vectors are defined in a natural way for homeomorphisms of $T^{2}$ (see [F], for example), but these sets can be quite different as the following simple example shows. In the below pictured homeomorphism of the torus there are two invariant circles, each one-sided attracting and one-sided repelling, one with rotation number 0 and the other with rotation number $\frac{1}{2}$. Then $\rho_{\psi}=\left\{(0, t) \mid t \in\left[0, \frac{1}{2}\right]\right\}$ while $\rho=\left\{(0,0),\left(0, \frac{1}{2}\right)\right\}$.

In preparation for the proof of Theorem 3.2 we prove a sequence of lemmas.

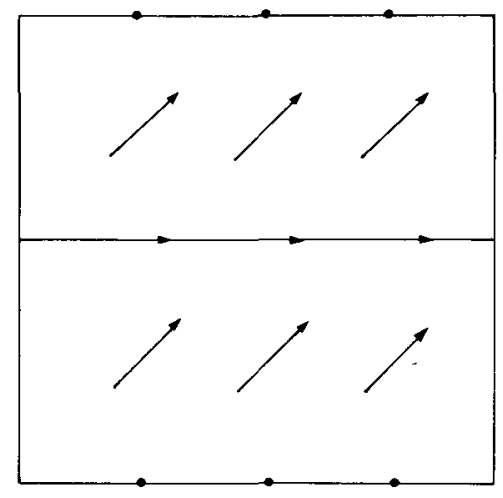

FIGURE 1 
LeMmA 3.3. Let $f: A \rightarrow A$ be a homeomorphism with lift $F: \tilde{A} \rightarrow \tilde{A}$ and let $T: \tilde{A} \rightarrow \tilde{A}$ be the deck transformation $T(x, y)=(x+1, y)$. Let $p$ and $q$ be positive integers and suppose that $\tilde{Z}$ is the lift of an invariant set $Z$. Then

(i) $\rho_{\psi}\left(T^{-p} F^{q} \mid \dot{z}\right)=q \cdot \rho_{\psi}(F \mid \tilde{z})-p$ and

(ii) $\rho\left(T^{-p} F^{q} \mid \tilde{z}\right)=q \cdot \rho(F \mid \tilde{z})-p$.

Proof. This is essentially Lemma 1.1 of Boyland [Bd]. We leave the obvious modifications to the reader.

LEMMA 3.4. Let $Z$ be compact and invariant under the homeomorphism $f: A \rightarrow A$. Then $\left\langle\rho_{\psi}(Z)\right\rangle=\langle\rho(Z)\rangle$.

Proof. Clearly $\langle\rho(Z)\rangle \subset\left\langle\rho_{\psi}(Z)\right\rangle$. Let $p / q$ be a rational with sup $\rho(Z)<p / q$ and let $G=T^{-p} F^{q}$. Then $G$ is a lift of $f^{q}$ with sup $\rho\left(\left.G\right|_{\tilde{Z}}\right)<0$ by Lemma 3.3. By compactness of the unit square intersected with $\bar{Z}$ there is an integer $N$ such that, for some $\gamma>0, G^{N}\left(S_{1} \cap \tilde{Z}\right) \subset S_{-1-\gamma}$ where $S_{r}$ is the half-strip $S_{r}=\{z \mid \pi \tilde{z} \leq r\}$. Periodicity now yields

(1) $G^{k N}\left(S_{1} \cap \tilde{Z}\right) \subset S_{-k-\gamma}$ for $k=1,2, \ldots$

Now let $\left\{\tilde{z}_{n}\right\}$ be an $\alpha$-pseudo-orbit for $G$ which is the lift of an $\alpha$-pseudo-orbit for $f^{a}$ that lies in $Z$. For sufficiently small $\alpha>0,\left\{\tilde{z}_{k N}\right\}_{k=0}^{\infty}$ is a $\gamma$-pseudo-orbit for $G^{N}$ and, from (1), we conclude that

(2) $\tilde{z}_{k N} \in S_{-k}$ for $k=1,2, \ldots$.

By continuity there is an $M>0$ such that if $\tilde{z}_{n} \in S_{-M}$ then $\tilde{z}_{n+j} \in S_{0}$ for $j=$ $0,1, \ldots, N-1$. Thus we see that $\tilde{z}_{n} \in S_{0}$ for all sufficiently large $n$ and $\sup \rho\left(\left\{\tilde{z}_{n}\right\}\right) \leq 0$. Since the $\alpha$-pseudo orbit was arbitrary, it follows that $\sup \rho_{\psi}\left(\left.G\right|_{\tilde{z}}\right) \leq 0$ which, by Lemma 3.3, implies that $\sup \rho_{\psi}\left(\left.F\right|_{\tilde{Z}}\right) \leq p / q$. Since $p / q>\sup \rho(Z)$ was arbitrary, $\sup \rho_{\psi}(Z) \leq \sup \rho(Z)$. A similar argument shows that inf $\rho(Z) \leq \inf \rho_{\psi}(Z)$. Finally, $\inf \rho(Z) \in \rho(Z)$ and $\sup \rho(Z) \in \rho(Z)$ by Theorem 2.5 so that $\inf \rho(Z)=\inf \rho_{\psi}(Z)$ and $\sup \rho(Z)=\sup \rho_{\psi}(Z)$.

We will employ the following terminology. Given a sequence of sets $S_{n}$, let

$$
\limsup _{n \rightarrow \infty} S_{n}=\bigcap_{N \geq 0} c l\left(\bigcup_{n=N}^{\infty} S_{n}\right)
$$

and, given a sequence of points $x_{m}$, let $\omega\left(\left\{x_{m}\right\}\right)=\lim \sup _{n \rightarrow \infty} S_{n}$ with $S_{n}=$ $\left\{x_{m} \mid m \geq n\right\}$. A set $S$ in $A$ will be called $\beta$-invariant (under the homeomorphism $f$ ) if $\mathrm{d}(f(x), S) \leq \beta$ for all $x \in S$ and we will say that $S$ is $\beta$-chain transitive if, given $x, y \in S$, there are points $x=x_{0}, x_{1}, \ldots, x_{n}=y$ in $S$ such that

$$
d\left(f\left(x_{i}\right), x_{i+1}\right) \leq \beta \text { for } i=0, \ldots, n-1 .
$$

Lемма 3.5. If $\left\{x_{n}\right\}_{n=0}^{\infty}$ is an $\alpha$-pseudo-orbit for the homeomorphism $f: A \rightarrow A$ then $\omega\left(\left\{x_{n}\right\}\right)$ is $\alpha$-invariant and $\beta$-chain transitive for all $\beta>\alpha$.

Proof. Let $x \in \omega\left(\left\{x_{n}\right\}\right)$. Then there is a subsequence $x_{n_{i}}$ with $x_{n_{i}} \rightarrow x$. Then $f\left(x_{n_{i}}\right) \rightarrow f(x)$ and, since $d\left(f\left(x_{n_{i}}\right), x_{n_{i}+1}\right) \leq \alpha$, the accumulation points of the $x_{n_{i}+1}$ are in $\omega\left(\left\{x_{n}\right\}\right)$ and within $\alpha$ of $f(x)$. Thus $\omega\left(\left\{x_{n}\right\}\right)$ is $\alpha$-invariant.

Now let $x, y \in \omega\left(\left\{x_{n}\right\}\right)$ and let $\varepsilon>0$ be given. Choose $0<\delta<\varepsilon$ so that $d(z, w)<\delta$ implies $d(f(z), f(w))<\varepsilon$, choose $N$ large enough so that $d\left(x_{n}, \omega\left(\left\{x_{n}\right\}\right)\right)<\delta$ for all 
$n \geq N$, and let $k$ and $m$ be larger than $N$ with $d\left(x_{k}, x\right)<\delta, d\left(x_{m}, y\right)<\varepsilon$, and $m \geq k$. Let $n=m-k$, let $z_{0}=x, z_{n}=y$ and, for $i=1, \ldots, n-1$, choose $z_{i} \in \omega\left(\left\{x_{n}\right\}\right)$ such that $d\left(z_{i}, x_{k+i}\right)<\delta$. It is easy to check that $x=z_{0}, z_{1}, \ldots, z_{n-1}, z_{n}=y$ is an $\alpha+2 \varepsilon$ chain in $\omega\left(\left\{x_{n}\right\}\right)$ from $x$ to $y$.

Given a homeomorphism $f: A \rightarrow A$, an arbitrary subset $S \subset A$, and $\alpha>0$, let $\rho(S, \alpha)=\bigcup \rho\left(\left\{x_{n}\right\}\right)$, the union being over all $\alpha$-pseudo-orbits $\left\{x_{n}\right\}_{n=0}^{\infty}$ of $f$ lying entirely in $S$.

LeMmA 3.6. Let $f: A \rightarrow A$ be a homeomorphism and let $S_{n} \subset A, n=0,1,2, \ldots$ be a sequence of sets with the properties: (i) there is a convergent sequence $\left\{x_{n}\right\}_{n=0}^{\infty}$ with $x_{n} \in S_{n}$ for $n=0,1,2, \ldots$ and (ii) $S_{n}$ is $\alpha_{n}$-invariant and $\alpha_{n}$-chain transitive for each $n$ with $\alpha_{n} \rightarrow 0$ as $n \rightarrow \infty$. Then $S=\limsup _{n \rightarrow \infty} S_{n}$ is compact invariant and chain transitive. Furthermore, $\lim \sup _{n \rightarrow \infty}\left(S_{n}, \alpha_{n}\right) \subset \rho_{\psi}(S)$.

Proof. Of course $S$ is compact. Let $z \in S$ and choose $z_{i} \in S_{n_{i}}$ with $z_{i} \rightarrow z$. Then $f\left(z_{i}\right) \rightarrow f(z)$ and there are $w_{i} \in S_{n_{i}}$ with $d\left(w_{i}, f\left(z_{i}\right)\right)<\alpha_{n_{i}}+1 / i$. Then $w_{i} \rightarrow f(z)$ so $f(z) \in S$ and $S$ is invariant.

Now let $x=\lim _{n \rightarrow \infty} x_{n} \in S$, let $y$ be any other element of $S$ and let $\alpha$ be greater than zero. Let $\delta>0$ be small enough so that $\delta<\alpha / 3$ and, if $d(z, w)<\delta$, then $d(f(z), f(w))<\alpha / 3$. Choose $m$ large enough so that $d\left(x_{m}, x\right)<\delta$, so that if $w \in S_{m}$ then $d(w, S)<\delta$, and such that $\alpha_{m}<\alpha / 3$. We may also choose such an $m$ so that there is a $y_{m} \in S_{m}$ with $d\left(y_{m}, y\right)<\alpha / 3$.

Now in $S_{m}$, let $w_{0}=x_{m}, w_{1}, w_{2}, \ldots, w_{n}=y_{m}$ be an $\alpha_{m}$-chain from $x_{m}$ to $y_{m}$ and select $z_{0}=x, z_{1}, \ldots, z_{n}=y$ in $S$ with $d\left(z_{i}, w_{i}\right)<\delta$ for $i=0, \ldots, n$. Then

$$
\begin{aligned}
d\left(f\left(z_{i}\right), z_{i+1}\right) & \leq d\left(f\left(z_{i}\right), f\left(w_{i}\right)\right)+d\left(f\left(w_{i}\right), w_{i+1}\right)+d\left(w_{i+1}, z_{i+1}\right) \\
& \leq \alpha / 3+\alpha / 3+\alpha / 3=\alpha \text { so } z_{0}, z_{1}, \ldots, z_{n}
\end{aligned}
$$

is an $\alpha$-chain from $x$ to $y$ in $S$. Clearly, using the same $S_{m}$, we can also construct an $\alpha$-chain from $y$ to $x$. Thus, given $\alpha>0$ and any $y, z \in S$ we can find an $\alpha$-chain in $S$ (going through $x$ ) from $y$ to $z$ so $S$ is chain transitive.

We now show that given $\alpha>0$ there is an $N$ such that $\rho\left(S_{n}, \alpha_{n}\right) \subset \rho(S, \alpha)$ for all $n \geq N$. From this it will follow that

$$
\limsup _{n \rightarrow \infty} \rho\left(S_{n}, \alpha_{n}\right) \subset \bigcap_{\alpha>0} \rho(S, \alpha)=\rho_{\psi}(S) .
$$

To this end let $\alpha>0$ be given. Let $\delta$ be small enough so that $\delta \leq \alpha / 3$ and, if $d(\tilde{z}, \tilde{w})<\delta$ then $d(F(\tilde{z}), F(\tilde{w}))<\alpha / 3$. Now take $N$ large enough so that $d(w, S)<\delta$ for all $w \in \bigcup_{n \geq M} S_{n}$ and $\alpha_{n} \leq \alpha / 3$ for all $n \geq N$. Now let $n$ be at least as large as $N$ and let $\left\{\tilde{w}_{k}\right\}_{k=0}^{\infty}$ be an $\alpha_{n}$-pseudo-orbit in $\tilde{S}_{n}$. Choose $\tilde{z}_{k} \in \tilde{S}$ such that $d\left(\tilde{z}_{k}, \tilde{w}_{k}\right)<\delta$. Then

$$
\begin{aligned}
d\left(F\left(\tilde{z}_{k}\right), \tilde{z}_{k+1}\right) & \leq d\left(F\left(\tilde{z}_{k}\right), F\left(\tilde{w}_{k}\right)\right)+d\left(F\left(\tilde{w}_{k}\right), \tilde{w}_{k+1}\right)+d\left(\tilde{w}_{k+1}, \tilde{z}_{k+1}\right) \\
& <\alpha / 3+\alpha / 3+\alpha / 3=\alpha \quad \text { for } k=0,1, \ldots,
\end{aligned}
$$

and we see that $\left\{\tilde{z}_{k}\right\}$ is an $\alpha$-pseudo-orbit in $\tilde{S}$. Since $d\left(\tilde{z}_{k}, \tilde{w}_{k}\right)<\delta$ for all $k$, $\rho\left(\left\{\tilde{z}_{k}\right\}\right)=\rho\left(\left\{\tilde{w}_{k}\right\}\right)$ and we have $\rho\left(S_{n}, \alpha_{n}\right) \subset \rho(S, \alpha)$. 
Lemma 3.7. Let $f: A \rightarrow A$ be a homeomorphism isotopic to the identity. Given $l \in$ $c l(\rho(f))$ there is a sequence $l_{n} \in \rho(f)$ with $\lim _{n \rightarrow \infty} l_{n}=l$ and a sequence of compac invariant chain transitive sets $S_{n} \in A$ with $l_{n} \in \rho\left(S_{n}\right)$.

Proof. First suppose that there is a sequence of rationals $l_{n} \in \rho(f)$ with $l_{n} \rightarrow l$. For each $n$, let $z_{n} \in A$ with $l_{n} \in \rho\left(z_{n}\right)$. Then $l_{n} \in\left\langle\rho\left(\omega\left(z_{n}\right)\right)\right\rangle$ (see, for example, [Bd], Lemma $1.1)$ and, since $\omega\left(z_{n}\right)$ is a compact invariant chain transitive set, there is, by Theorem 3.1, a periodic orbit $S_{n}$ with rotation number $l_{n}$.

Now, if there is no sequence of rationals $l_{n} \rightarrow l, l_{n} \in \rho(f)$, then there must be a sequence of irrationals $l_{n} \rightarrow l, l_{n} \in \rho(f)$, and corresponding points $z_{n} \in A$ with $\rho\left(\omega\left(z_{n}\right)\right)=\left\{l_{n}\right\}$. Indeed, if for each sequence $z_{n} \in A$ with $l_{n} \in \rho\left(\omega\left(z_{n}\right)\right)$ there are infinitely many $n$ with $\rho\left(\omega\left(z_{n}\right)\right)$ containing more than $l_{n}$, then for each such $n$, there is a rational in $\rho(f)$ arbitrarily close to $l_{n}$ and we would have a sequence of rationals in $\rho(f)$ converging to $l$. Thus, in this case, we may let $S_{n}=\omega\left(z_{n}\right)$.

Lемма 3.8. Let $\left\{z_{n}\right\}$ be an $\alpha$-pseudo-orbit for the homeomorphism $f: A \rightarrow A$. Then $\rho\left(\omega\left(\left\{z_{n}\right\}\right), \beta\right) \supset \rho\left(\left\{z_{n}\right\}\right)$ for all $\beta>\alpha$.

Proof. For $\delta>0$ let $M=M(\delta)$ be such that $d\left(\tilde{z}_{m}, \omega\left(\left\{z_{n}\right\}\right)\right)<\delta$ for all $m \geq M$. For each $k=0,1,2, \ldots$ let $w_{k} \in \omega\left(\left\{z_{n}\right\}\right)$ be such that $d\left(z_{M+k}, w_{k}\right)<\delta$. Clearly for a given $\beta>\alpha$, there is a $\delta>0$ small enough so that $w_{k}$ is a $\beta$-pseudo-orbit in $\omega\left(\left\{z_{n}\right\}\right)$. Since $d\left(\tilde{w}_{n}, \tilde{z}_{n}\right)$ is bounded, $\rho\left(\left\{\tilde{w}_{n}\right\}\right)=\rho\left(\left\{\tilde{z}_{n}\right\}\right)$ so that $\rho\left(\left\{z_{n}\right\}\right) \subset \rho\left(\omega\left(\left\{z_{n}\right\}\right), \beta\right)$.

LEMMA 3.9. If $Z$ is a compact invariant chain transitive set for the homeomorphism $f: A \rightarrow A$ then $\rho_{\psi}(Z)=\left\langle\rho_{\psi}(Z)\right\rangle$ is a closed interval.

Proof. According to Lemma 3.4, $\left\langle\rho_{\psi}(Z)\right\rangle=\langle\rho(Z)\rangle$ and, by Theorem $2.5,\langle\rho(Z)\rangle$ is a closed interval, say $[a, b]$, the endpoints of which are realized as actual rotation numbers of elements of $Z$. Let $x, y \in Z$ be such that $\rho(x)=a$ and $\rho(y)=b$.

Now let $\alpha>0$ be given. By compactness and chain transitivity of $Z$ there is an $N=N(\alpha)$ such that, given any $w, z \in Z$ there is an $\alpha$-chain for $f$ from $w$ to $z$ of length no larger than $N$. Thus, for any sequence of positive integers $0=$ $N_{0}, N_{1}, \ldots$ with $N_{k+1}-N_{k}>N$ for $k=0,1, \ldots$, there is an $\alpha$-pseudo-orbit $\left\{\tilde{z}_{n}\right\}$ for $F$ with the property that, for $n \in\left[N_{k}, N_{k+1}-N\right], \tilde{z}_{n}$ is a true orbit segment of $F, \tilde{z}_{n}$ is on the orbit of $\tilde{x}$ for $k$ even, and $\tilde{z}_{n}$ is on the orbit of $\tilde{y}$ for $k$ odd. Now since $\rho(\tilde{x})=a$ and $\rho(\tilde{y})=b$ it is clear that, by letting $N_{k+1}$ be large enough with respect to $N_{k}$, we will have

$$
\pi\left(\tilde{z}_{N_{k+1}}\right) / N_{k+1}<a+\frac{1}{k+1} \text { for } k \text { even }
$$

and

$$
\pi\left(\tilde{z}_{N_{k+1}}\right) / N_{k+1}>b-\frac{1}{k+1} \text { for } k \text { odd. }
$$

Then $a, b \in \rho\left(\left\{z_{n}\right\}\right)$ so that (see [B-M-P-T]) $[a, b] \subset \rho\left(\left\{z_{n}\right\}\right)$. Thus $\left\langle\rho_{\psi}(Z)\right\rangle=$ $[a, b] \subset \rho(Z, \alpha)$ for all $\alpha>0$ and so $\rho_{\psi}(Z)=\left\langle\rho_{\psi}(Z)\right\rangle$.

Proof of Theorem 3.2. Given an $l$ in $c l(\rho(f))$ there are sequences $l_{n}$ in $\rho(f)$ and compact invariant chain transitive sets $S_{n} \subset A$ such that $l_{n} \rightarrow l$ and $l_{n} \in \rho\left(S_{n}\right)$ (Lemma 
3.7). We may assume there is a sequence $x_{n} \in S_{n}$ such that $\left\{x_{n}\right\}$ is convergent (otherwise, take a subsequence of the $S_{n}$ 's). Let $\alpha_{n}=1 / n$. Then, by Lemma 3.6,

$$
l \in \limsup _{n \rightarrow \infty} \rho\left(S_{n}, \alpha_{n}\right) \subset \rho_{\psi}\left(\limsup _{n \rightarrow \infty} S_{n}\right) .
$$

In particular, $l \in \rho_{\psi}(f)$.

Now let $l$ be an element of $\rho_{\psi}(f)$. Let $\alpha_{n}>0$ be a sequence with $\alpha_{n} \rightarrow 0$ as $n \rightarrow \infty$. Since $l \in \rho_{\psi}(f)$ there is, for each $n$, an $\alpha_{n}$-pseudo-orbit $\left\{x_{k}^{n}\right\}_{k=0}^{\infty}$ with $l \in \rho\left(\left\{x_{k}^{n}\right\}\right)$. Let $S_{n}=\omega\left(\left\{x_{k}^{n}\right\}_{k=0}^{\infty}\right)$. Then, by Lemma 3.8, $l \in \rho\left(S_{n}, \beta_{n}\right)$ for $\beta_{n}=\alpha_{n}+1 / n, S_{n}$ is $\beta_{n}$-chain transitive and $\beta_{n}$-invariant for all $n$ (Lemma 3.5). By passing to a subsequence if necessary we may assume that there is a convergent sequence $\left\{x_{n}\right\}$ with $x_{n} \in S_{n}$ for all $n$. By Lemma 3.6, $l \in \rho_{\psi}(S)$ where $S=\lim _{\sup _{n \rightarrow \infty}} S_{n} . S$ is a compact invariant chain transitive set and $\rho_{\psi}(S)=\left\langle\rho_{\psi}(S)\right\rangle=\langle\rho(S)\rangle$ by Lemmas 3.4 and 3.9. According to Theorem 3.1, every rational in $\langle\rho(S)\rangle$ is in $\rho(f)$. Thus $l \in c l\langle\rho(S)\rangle$ so $l \in c l(\rho(f))$.

Corollary 3.10. If $f: A \rightarrow A$ is a positive tilt diffeomorphism then $\rho(f)=\rho_{\psi}(f)$.

Proof. For such an $f, \rho(f)$ is closed ([Bd]).

It is apparently unknown whether $\rho(f)$ is always closed and if all elements of $\rho(f)$ are realized as rotation numbers. The following proposition provides some information regarding this.

Proposition 3.11. Let $f: A \rightarrow A$ be a homeomorphism isotopic to the identity. If $l \in c l(\rho(f)) \cap c l\left(\operatorname{int}\left((\rho(f))^{c}\right)\right)$ or if $l$ is rational and in $c l(\rho(f))$ then $l$ is the rotation number of some point in $A$. In fact, for such $l$, there is an invariant measure $\mu$ such that $\rho(z)=l$ for $\mu$-almost all $z \in A$.

Proof. Given an $l$ as above, the existence of a $z \in \Lambda$ with $\rho(z)=l$ can be obtained from Theorems 2.5 and 3.1 and from the lemmas used in the proof of Theorem 3.2. the following alternative may be more enlightening.

Let $l \in c l(\rho(f))$. Then, by Lemma 3.7, there is a sequence $l_{n} \in \rho(f)$ and compact invariant chain transitive sets $S_{n} \subset A$ such that $l_{n} \rightarrow l$ and $l_{n} \in \rho\left(S_{n}\right)$. In fact we may assume $l_{n}=\rho\left(S_{n}\right)$ since if $\rho\left(S_{n}\right)$ is more than a single number we can replace $l_{n}$ by an arbitrarily close rational in $\left\langle\rho\left(S_{n}\right)\right\rangle$ and replace $S_{n}$ by a periodic orbit having that rational as its rotation number (Theorem 3.1).

Now let $\mu_{n}$ be an ergodic invariant measure supported on $S_{n}$ (see, for example, [D-G-S]). By taking subsequences if necessary we may assume that the $\mu_{n}$ converge to the invariant measure $\mu$ in the weak ${ }^{*}$-topology and that there is a convergent sequence $\left\{x_{n}\right\}$ with $x_{n} \in S_{n}$. Then, by Lemma 3.6, $l \in \rho(S)$ where $s=\lim \sup _{n \rightarrow \infty} S_{n}$ is a compact invariant chain transitive set. Also, it is not hard to see that the support of $\mu$ is in $S$. Now let $g=\pi \circ(F-$ Id $) \circ \sigma$ as in $\S 2$ and let

$$
g^{*}(z)=\lim _{n \rightarrow \infty} \frac{1}{n} \sum_{k=0}^{n} g\left(f^{k}(z)\right)
$$

since $g$ is continuous and $\mu_{n} \rightarrow \mu$ in the weak*-topology we have $\int g d \mu_{n} \rightarrow \int g d \mu$. By the ergodic theorem, $\int g d \mu_{n}=g^{*}(z)$ for $\mu_{n}$ almost all $z$. Since $\mu_{n}$ is supported 
on $S_{n}$ and $\rho\left(S_{n}\right)=l_{n}$, we have (Proposition 2.3) $g^{*}(z)=l_{n}$ for $\mu_{n}$-almost all $z$. Thus $\int g d \mu=l$.

Now suppose that $g^{*}(z) \neq l$ on a set of positive measure with respect to $\mu$. Then $\int g^{*} d \mu=l$ by Proposition 2.3 and there are $z_{1}, z_{2} \in S$ such that $g^{*}\left(z_{1}\right)<l$ and $g^{*}\left(z_{2}\right)>l$. Since $g^{*}\left(z_{1}\right)$ and $g^{*}\left(z_{2}\right)$ are rotation numbers, we have that $l$ is in the interior of the nontrivial interval $\operatorname{cl}\langle\rho(S)\rangle$. But $c l(\langle\rho(S)\rangle) \subset c l(\rho(f))$ by Theorem 3.1 so $l \notin c l\left(\right.$ int $\left.\left((\rho(f))^{c}\right)\right)$.

Thus for $l \in c l(\rho(f)) \cup c l\left(\right.$ int $\left.\left((\rho(f))^{c}\right)\right)$ we have $g^{*}(z)=l$ for $\mu$-almost all $z \in A$. That is, $\rho(z)=l$ for $\mu$-almost all $z \in A$.

We leave the argument for $l$ a rational in $c l(\rho(f))$ to the reader.

Corollary 3.12. Let $f: A \rightarrow A$ be a homeomorphism isotopic to the identity. If each connected component of $\rho(f)$ has positive length or is isolated from the other components then $\rho(f)$ is closed.

In $\S 1$ it was shown that circle endomorphisms have the rotation shadowing property. This is certainly not the case for all homeomorphisms of the annulus. Consider, for example, a homeomorphism that leaves invariant all meridional circles and has a nontrivial rotation interval. Then, for each $\alpha$, there is an $\alpha$-pseudo-orbit having that interval as its rotation set but every true orbit has a rotation number. The following proposition gives another situation in which the rotation set is closed and, if Conjecture 1 at the end of this paper is correct, implies that, at least generically, the rotation set is closed.

Proposition 3.13. If $f: A \rightarrow A$ is a homeomorphism isotopic to the identity that has the rotation shadowing property then $\rho(f)$ is closed.

Proof. Let $l \in c l(\rho(f))$. Then (see the proof of Proposition 3.11) there is a compact invariant chain transitive set $S$ with $l \in\langle\rho(S)\rangle$. By Theorem $2.5,\langle\rho(S)\rangle$ is closed so if $l \in \partial\langle\rho(S)\rangle$ then $l \in \rho(f)$. If $l \in$ int $(\langle\rho(S)\rangle)$, let $\beta=\frac{1}{2} d(l, \partial\langle\rho(S)\rangle)$. Since $S$ is compact invariant and chain transitive, there is, for each $\alpha>0$, an $\alpha$-pseudo-orbit with rotation set containing $\langle\rho(S)\rangle$ (see the proof of Lemma 3.9). Let $\alpha$ be small enough so that every $\alpha$-pseudo-orbit is $\beta$-rotation shadowed by a true orbit. Then a true orbit $\beta$-rotation shadowing an $\alpha$-pseudo-orbit whose rotation set contains $\langle(S)\rangle$ has $l$ in its rotation interval. Thus, $l \in \rho(f)$.

For our final result we let $\Omega$ be the set of all homeomorphisms of the annulus isotopic to the identity and we let $H(R)$ denote the set of all closed subsets of the reals. We give $\Omega$ the $C^{0}$-topology and $H(R)$ the Hausdorf topology. Let $\bar{\rho}: \Omega \rightarrow H(R)$ be given by $\bar{\rho}(f)=c l(\rho(f))$.

THEOREM 3.14. $\bar{\rho}$ is upper semicontinuous. That is, if $f_{n} \in \Omega$ and $f_{n} \rightarrow f \in \Omega$ uniformly then $\lim \sup _{n \rightarrow \infty} \bar{\rho}\left(f_{n}\right) \subset \bar{\rho}(f)$.

Proof. Without loss of generality we may assume that $d\left(f_{n}, f\right)=\alpha_{n} \rightarrow 0$ monotonically. Then the $\alpha_{n}$-pseudo-rotation sets $\rho\left(f, \alpha_{n}\right)$ are nested and, since every orbit of $f_{n}$ is 
an $\alpha_{n}$-pseudo-orbit of $f, \rho\left(f_{n}\right) \subset \rho\left(f, \alpha_{n}\right)$. Thus,

$$
\begin{aligned}
\underset{n \rightarrow \infty}{\limsup } \bar{\rho}\left(f_{n}\right) & =\bigcap_{N \geq 0}\left(\bigcup_{n \geq N} \bar{\rho}\left(f_{n}\right)\right) \\
& \subset \bigcap_{n \geq 0} \operatorname{cl}\left(\rho\left(f, \alpha_{n}\right)\right) \\
& =\rho_{\psi}(f) \\
& =\bar{\rho}(f) .
\end{aligned}
$$

The last equality is Theorem 3.2.

Remark. Boyland [Bd] proves a version of the above theorem with $\Omega$ replaced by the positive tilt diffeomorphisms equipped with the $C^{1}$ topology. Since $C^{0}$ is weaker than $C^{1}$, Theorem 3.14 strengthens and extends Boyland's result.

Examples of $f \in \Omega$ at which $\bar{\rho}$ is not continuous abound - the example following Corollary 3.13 is one such - but, since $\Omega$ is a Baire space, the set on which $\bar{\rho}$ is continuous is generic ([Ch]).

We conclude with a few conjectures.

Conjecture 1. For a generic set of $f \in \Omega, f$ has the rotation shadowing property.

Conjecture 2. If $f \in \Omega$ then $\rho(f)$ is closed.

Conjecture 3. Given $f \in \Omega, \alpha>0$, and $l \in \rho_{\psi}(f)$, there is an $\alpha$-pseudo-orbit $\left\{z_{n}\right\}$ with $\rho\left(\left\{z_{n}\right\}\right)=l$.

Conjecture 4. Given $f \in \Omega$ and $l \in \rho(f)$, there is a $z \in A$ with $\rho(z)=l$.

\section{REFERENCES}

[Bi] G. D. Birkhoff. Sur l'existence de regions d'instabilite en dynamique. Ann. Inst. H. Poincaré 2 (also Collected Math. Papers) (1932).

[Bd] P. Boyland. Rotation Sets and Morse Decompositions in Twist Maps. Reprint (1986).

[B-M-P-T] R. Bamon et al. Rotation intervals of endomorphisms of the circle. Ergod. Th. \& Dynam. Sys. 4 (1984), 493-499.

[Bo] R. Bowen. On axiom A diffeomorphisms. CBMS Series No. 35 Amer. Math. Soc. (1978).

[Ch] G. Choquet. Lectures on Analysis, Vol. I, W. A. Benjamin: New York, 1969.

[D-G-S] M. Denker et al. Ergodic Theory of Compact Spaces. Lecture Notes in Mathematics, Vol. 527, Springer-Verlag: New York, 1976.

[F] J. Franks. Recurrence and fixed points of surface homeomorphisms. To appear in Ergod. Th. \& Dynam. Sys.

[Ha] G. R. Hall. A topological version of a theorem of Mather on twist maps. Ergod. Th. \& Dynam. Sys. 4 (1984), 585-603.

[He] M. Herman. Sur les courbes invariantes par les difféomorphismes de l'anneau. Astérisque 103-104 (1983).

[I] R. Ito. Rotation sets are closed. Math. Proc. Camb. Phil. Soc. 89 (1981), 107-111.

[K] A. Katok. Some remarks on the Birkhoff and Mather Twist Theorems. Ergod. Th. \& Dynam. Sys. 2 (1982), 183-194.

[Ma] J. Mather. Non-existence of invariant circles. Ergod. Th. \& Dynam. Sys. 4 (1984), 301-309.

[N-P-T] S. Newhouse et al. Bifurcations and stability of families of diffeomorphisms. IHES Publ. Math. 57 (1983), 5-71.

[R] W. Rudin. Functional Analysis, McGraw-Hill: New York, 1973. 